\title{
GORENSTEIN ALGEBRAS WITH NONUNIMODAL BETTI NUMBERS
}

\author{
MATS BOIJ
}

\begin{abstract}
We show the existence of graded Gorenstein algebras whose Betti numbers are not unimodal, contradicting a conjecture by R. Stanley. In fact, we prove that the Betti numbers given by the natural lower bound are non-unimodal in sufficiently high embedding dimension - greater than or equal to nine - and we have calculated two examples where the Betti numbers attain this lower bound.
\end{abstract}

The Hilbert function of graded Gorenstein algebras were once conjecured to be unimodal. R. Stanley [7, Ex. 4.3] found a counterexample and later large classes of counterexamples have been found by others $[2,4,5]$.

Later R. Stanley [8, Conj. 4b] conjectured that at least the Betti numbers of a graded Gorenstein algebra should be unimodal. In many examples this is also the case. For example it is clear that the Betti numbers of a complete intersection are unimodal.

In a previous paper [3], we stated a conjecture on the generic Betti numbers of compressed Gorenstein Artin algebras. In this paper we prove that our conjecture implies that the Betti numbers of a sufficiently general Gorenstein Artin algebra of embedding dimension at least 9 are non-unimodal if the degree of the socle is odd, contrary to the conjecture by Stanley.

This, of course, does not prove the existence of Gorenstein algebras with non-unimodal Betti numbers. Therefore we have, by means of the computer algebra system Macaulay [1], computed the Betti numbers for two examples of graded Gorenstein algebras, with embedding dimension 9 and 10, where the Betti numbers are non-unimodal.

Setur 1. Fix integers $r$ and $c$. Let $R$ be the polynomial ring $k\left[x_{1}, x_{2}, \ldots, x_{r}\right]$ over a field $k$ of characteristic 0 . For any hyperplane $H \subseteq R_{c}$ we define $(H: R)_{d}=\left\{a \in R_{d} \mid a b \in H\right.$, for all $\left.b \in R_{c-d}\right\}$. The quotient $A=R / \bigoplus_{d \geq 0}(H: R)_{d}$ is then a Gorenstein Artin algebra with socle in degree $c$ [5, Prop. 2.4]. If $H$ is sufficiently general in $R_{d}$, we have that $A$ is com-

Received December 17, 1996. 
pressed, which means that the Hilbert function of $A$ is $\min \left\{\operatorname{dim}_{k} R_{d}, \operatorname{dim}_{k} R_{c-d}\right\}$ - the maximal possible (cf. [6, Thm. I] and [5, Thm. 3.4]).

Definition 2. For any two positive integers $r$ and $c$ we define a sequence of numbers $b_{0}, b_{1}, \ldots, b_{r}$ by $b_{i}=b_{i}^{\prime}+b_{i}^{\prime \prime}$, for $i=1,2, \ldots, r-1$, where

$$
\begin{aligned}
& b_{i}^{\prime}=\max \left\{0,\left(\begin{array}{c}
t-1+i-1 \\
i-1
\end{array}\right)\left(\begin{array}{c}
t-1+r \\
r-i
\end{array}\right)-\left(\begin{array}{c}
c-t+r-i \\
r-i
\end{array}\right)\left(\begin{array}{c}
c-t+r \\
i-1
\end{array}\right)\right\} \\
& b_{i}^{\prime \prime}=\max \left\{0,\left(\begin{array}{c}
c-t+r-i-1 \\
r-i-1
\end{array}\right)\left(\begin{array}{c}
c-t+r \\
i
\end{array}\right)-\left(\begin{array}{c}
t-1+i \\
i
\end{array}\right)\left(\begin{array}{c}
t-1+r \\
r-i-1
\end{array}\right)\right\},
\end{aligned}
$$

for $i=1,2, \ldots, r-1$, where $t=\lfloor c / 2\rfloor+1$.

Conjecture 3 ([3, Conj. 4.23]). Suppose that $H$ is a sufficiently general hyperplane in $R_{c}$ and let $A=R / \bigoplus_{d \geq 0}(H: R)_{d}$. Then the Betti numbers of $A$ are given by $b_{0}, b_{1}, \ldots, b_{r}$, from Definition 2 .

Remark 4. In fact, the numbers given by the sequence $b_{0}, b_{1}, \ldots, b_{r}$ is a lower bound for the Betti numbers of any compressed Gorenstein Artin algebra of embedding dimension $r$ and socle in degree $c$ [3, §4-4].

Proposition 5. The sequence of numbers $1=b_{0}, b_{1}, b_{2}, \ldots, b_{r-1}, b_{r}=1 \mathrm{gi}$ ven by Definition 2 is non-unimodal if $c$ is odd and $r \geq 9$

Proof. The sequence $b_{0}, b_{1}, \ldots, b_{r}$ is symmetric around $\lfloor r / 2\rfloor$, i.e. satisfy $b_{i}=b_{r-i}$, for $i=0,1, \ldots, r$ and $b_{0}=b_{r}=1$. Hence it suffices to show that $b_{k-1}>b_{k}$, if $c$ is odd and $r \geq 9$, where $k=\lfloor r / 2\rfloor$.

We first consider the case where $r=2 k+1$. We have that $c=2 t-1$ where $t$ is the least integer such that $\left(\begin{array}{c}r-1+t \\ r-1\end{array}\right)>\left(\begin{array}{c}r-1+c-t \\ c-t\end{array}\right)$. We have now that the conjectured Betti numbers are

$$
\begin{aligned}
b_{k} & =\left(\begin{array}{c}
t+k-2 \\
k-1
\end{array}\right)\left(\begin{array}{c}
t+2 k \\
k+1
\end{array}\right)-\left(\begin{array}{c}
c-t+k+1 \\
k+1
\end{array}\right)\left(\begin{array}{c}
c-t+2 k+1 \\
k-1
\end{array}\right) \\
& =\frac{2(t+k)(t+k-2) !(t+2 k) !}{(t-1) !(k-1) !(k+1) !(t+k+1) !}
\end{aligned}
$$

and

$$
\begin{aligned}
b_{k-1} & =\left(\begin{array}{c}
t+k-3 \\
k-2
\end{array}\right)\left(\begin{array}{c}
t+2 k \\
k+2
\end{array}\right)-\left(\begin{array}{c}
c-t+k+2 \\
k+2
\end{array}\right)\left(\begin{array}{c}
c-t+2 k+1 \\
k-2
\end{array}\right) \\
& =\frac{4(t+k+1)(t+k)(t+k-1)(t+2 k) !(t+k-3) !}{(t-1) !(k-2) !(k+2) !(t+k+2) !} .
\end{aligned}
$$

Thus we have that 


$$
\begin{aligned}
\frac{b_{k-1}}{b_{k}} & =\frac{2(k-1)(t+k+1)(t+k-1)}{(k+2)(t+k+2)(t+k-2)} \\
& =\frac{2(k-1)}{k+2} \frac{(t+k)^{2}-1}{(t+k)^{2}-4}>\frac{2(k-1)}{k+2} \geq 1,
\end{aligned}
$$

for $k \geq 4$. Hence we have proved that the sequence is non-unimodal in the case $r=2 k+1$, for $k \geq 4$.

Now assume that $r=2 k$. Then we have that $b_{k}=b_{k}^{\prime}+b_{k}^{\prime \prime}=2 b_{k}^{\prime}$, since $b_{k}^{\prime}=b_{k}^{\prime \prime}$ from (2.1). Hence we have that

$$
\begin{aligned}
b_{k} & =2\left(\left(\begin{array}{c}
t-1+k-1 \\
k-1
\end{array}\right)\left(\begin{array}{c}
t-1+2 k \\
k
\end{array}\right)-\left(\begin{array}{c}
t-1+k \\
k
\end{array}\right)\left(\begin{array}{c}
t-1+2 k \\
k-1
\end{array}\right)\right) \\
& =\frac{2(t+k-2) !(t+2 k-1) !}{(t-1) ! k !(k-1) !(t+k) !}
\end{aligned}
$$

and

$$
\begin{aligned}
b_{k-1} & =\left(\begin{array}{c}
t-1+k-2 \\
k-2
\end{array}\right)\left(\begin{array}{c}
t-1+2 k \\
k+1
\end{array}\right)-\left(\begin{array}{c}
t-1+k+1 \\
k+1
\end{array}\right)\left(\begin{array}{c}
t-1+2 k \\
k-2
\end{array}\right) \\
& =\frac{3(t+k)(t+k-1)(t+k-3) !(t+2 k-1) !}{(t-1) !(k-2) !(k+1) !(t+k+1) !}
\end{aligned}
$$

Hence we have that

$$
\frac{b_{k-1}}{b_{k}}=\frac{3(k-1)(t+k-1)(t+k)}{2(k+1)(t+k+1)(t+k-2)}>\frac{3(k-1)}{2(k+1)} \geq 1,
$$

for $k \geq 5$. We have now finished the case $r=2 k$, for $k \geq 5$. Hence the sequence is non-unimodal if $r$ is odd and $r \geq 9$ or if $r$ is even and $r \geq 10$, which together finish the proof.

Remark 6. Proposition 5 shows that if Conjecture 3 holds we have that almost all graded Artin Gorenstein algebras with odd socle degree have nonunimodal Betti numbers if the number of variables is at least 9 .

For Gorenstein Artin algebrasof embedding dimension 9 and 10 with socle in degree 3 the Betti numbers given by Conjecture 3 are

$$
(1,36,160,315,288,288,315,160,36,1)
$$

$$
(1,45,231,550,693,660,693,550,231,45,1),
$$

respectively. Since it is a question of the generic case, it suffices to find one algebra having these Betti numbers to verify the conjecture (cf. $[3, \S \S 4-3$, 4-4]). The existence of such algebras has been verified by means of computer calculated resolutions. In in both cases the ideals are generated by quadrics. 
The algebras generated by the program were given by random codimension 1 vector spaces $H \subseteq R_{3}$ and the computer used $104 \mathrm{Mb}$ of memory to calculate the latter resolution. These Betti numbers are equal to the Betti numbers given by Conjecture 3 . Hence by the minimality of these Betti numbers there is no problem in the calculations being done in positive characteristic.

\section{REFERENCES}

1. D. Bayer and M. Stillman, Macaulay: A system for computation in algebraic geometry and commutative algebra, Source and object code available for Unix and Macintosh computers. Contact the authors, or download from zariski.harvard.edu via anonymous ftp.

2. D. Bernstein and A. Iarrobino, A nonunimodal graded Gorenstein Artin algebra in codimension five, Comm. Algebra 20 (1992), 2323-2336.

3. M. Boij, Artin level algebras, Ph.D. thesis, Royal Institute of Technology, Stockholm, 1994.

4. M. Boij, Graded Gorenstein Artin algebras whose Hilbert functions have a large number of valleys, Comm. Algebra 23 (1995), 97-103.

5. M. Boij and D. Laksov, Nonunimodality of graded Gorenstein Artin algebras, Proc. Amer. Math. Soc. 120 (1994), no. 4, 1083-1092.

6. A. Iarrobino, Compressed algebras: Artin algebras having given socle degrees and maximal length, Trans. Amer. Math. Soc. 285 (1984), 337-378.

7. R. Stanley, Hilbert functions of graded algebras, Adv. in Math. 28 (1978), 57-83.

8. R. Stanley, Log-concave and unimodal sequences in algebra, combinatorics, and geometry, Graph Theory and its Applications: East and West, Annals of the New York Acadamy of Sciences, vol. 576, The New York Acadamy of Scieces, 1989, pp. 500-535.

DEPARTMENT OF MATHEMATICS

$\mathrm{KTH}$

S-100 44 STOCKHOLM

SWEDEN

E-mail address: boij@math.kth.se 